\title{
Topological enhancement of split AC condenser brackets through CAE
}

\author{
B. Stalin ${ }^{1 *}$, J. Varun Siddharth ${ }^{2}$, G. Senthilkumar ${ }^{2}$ and M. Ravichandran ${ }^{3}$ \\ Assistant Professor, Department of Mechanical Engineering, Anna University, Regional Campus Madurai, \\ Keelakuilkudi, Madurai, Tamilnadu, India ${ }^{1}$ \\ PG Scholar, Department of Mechanical Engineering, Anna University, Regional Campus Madurai, Keelakuilkudi, \\ Madurai, Tamilnadu, India ${ }^{2}$ \\ Associate Professor, Department of Mechanical Engineering, K.Ramakrishnan College of Engineering, \\ Tiruchirappalli, Tamilnadu, India ${ }^{3}$
}

\section{(C2018 ACCENTS}

\begin{abstract}
For the development in research, depending upon the environment and concepts involved in design, new innovative ideas has become necessary for developing a better geometry in design for attaining a modified behavior in the analysis. While considering the design, it is often necessary for people involved in research, correlate both experimental and analytical design procedures. The percentage of similarity involved in both parameters of experimental and analytical design by the correlating analysis. It shows how far the design which is to be implemented is practically possible or not, even then if the design is practically possible, it is often necessary to consider cost to meet the product within an optimum budget. So the designer has to concentrate both in cost as well as the practicality feasible design. When we go through the past history in manufacturing or creating a product there is always a trouble which is to be considered as a major constraint which is known as weight. The optimal weight of the product is the major objective to be achieved. The computer aided engineering (CAE) technique has changed the strategy involved in research and development. Before this technique was introduced, people involved in research would change the geometry of the product based on the previous experience and similarities of the products. But this approach is time consuming, which can delay the progress of attaining an optimal value. This strategy would eliminate the time and cost involved in creating a prototype and testing them. Without finite element method the optimization processes could not be completed at an optimal time and so the finite element method model is the most suitable method for the optimization processes. In this paper, the product behavior for its topology of the split AC condenser bracket has been studied, where the weight of the bracket is reduced with enhanced safety factor through computer aided engineering.
\end{abstract}

\section{Keywords}

Split AC condenser bracket, Finite element analysis, Safety factor, CAE.

\section{Introduction}

The term topology in finite element analysis refers to network of mesh of definite size occupying a certain area or volume without disturbing the shape and size of the product keeping the product volume as constant. The model trend has changed the thoughts of the people to come up with easy handling equipment's and methods, reducing the mass of the product which is the major constraint in the industrial sectors. In addition the modification of the topology of the product has changed the aesthetics of the product which stimulates us to come up with new ideas and attract the customers.

\footnotetext{
*Author for correspondence
}

Al-Ketan et al. [1] studied the topological properties and its relationship for different classes of cellular materials is studied in order to understand the nature and the behavior of cellular materials in additive manufacturing.

Kazakis et al. [2] reported the possibilities of integrating the phase of intuition of civil structures along with automatic computational techniques in computer aided architectural design. Pleshakov [3] investigated the aid of theoretical techniques such as thermo fluctuation theory of fracture and graph theory the influence of mechanical properties of polymers in network topology in order to observe the nature of strength in the network of the polymers. Lin et al. [4] studied the topological nature of heat transferring material is observed through conduction in order to obtain the required heat transfer 
coefficient through topology optimization technique. Xingtong Yang and $\mathrm{Li}$ [5] studied the topological nature of the product of constant volume and mass with different materials as a mixture of compositions. $\mathrm{Xia}$ et al. [6] investigated the bidirectional evolutionary structural optimization method is used in order to minimize the stress in the design.

The major objective of this work is to optimize the bracket material layout in a confined design proportion without disturbing the design space under the given set of load and boundary conditions, for which the bracket model is to be experimented and experienced. As a result, the split AC condenser could be hoisted in wall with the bracket which will give a better topological performance, depending upon the load applied over it. Further the material density of the bracket model is reduced keeping its volume as constant.

The existing works on topology optimization were studied by many researchers. Based on the literature review, the work history of topology optimization is more important in order to obtain clarity in technical design strategy. This paper deals with reducing the topological density of the component in order to enhance the mechanical design features.

\section{Methodology}

\subsection{Static structural layout}

The term static means an object maintaining its stability or an object under neutral condition. The product which is to be studied for static analysis must have some fixed positions as well as the degree of freedom considering force or pressure in a definite space within its boundary conditions and constraints, the results which are obtained through static structural analysis are independent of time where the analytical event time interval is kept constant and the results are obtained within a specified time interval. There are many software tools which are used to study the static behavior and properties of the product like Hyper mesh, LS-Dyna, ANSYS, ABAQUS solver, Samcef etc. The static structural analysis through computer aided engineering tool helps us to come through solutions for various static analysis studies without giving an effort towards experimental strategy and wasting of time and money.
In many industries, the works involved for making prototypes has been reduced to a greater extent through Computer Aided Engineering.

\subsection{Load and boundary conditions}

The boundary condition is the application of a force and/or constraint. Figure 1 shows Load and boundary conditions of the split AC condenser bracket. Topology of split AC condenser bracket before refinement is presented in the Table 1 . The product which is supposed to be analyzed is imported into and analytical environment where degrees of freedom and loading conditions are created in order to observe the response of the product. When we consider for a bracket for analysis, the bracket is made to react like a cantilever beam with one end of the product is applied for degrees of freedom and the other end of the product is applied to force or loading conditions to attain the product deformation behavior at static condition is observed.

- The bracket which is to be analyzed is meshed for its material density.

- The fixtures are created at the circumference of the bracket hole; there are four bracket holes which are splitting into two pairs in a symmetrical manner.

- The load is applied as per the condenser weight. The load of $400 \mathrm{~N}$ which is approximately equal to $40 \mathrm{~kg}$ is applied uniformly over the horizontal flat surface of the bracket.

- The necessary results are solved using the workbench FEA solver and the solutions are obtained.

- The product results are evaluated for the required factor of safety.

- If the product is designed within the require factor of safety, then the analysis is completed, otherwise the above said procedures are repeated for varying iterations.

Table 1 Topology of split AC condenser bracket before refinement

\begin{tabular}{lll}
\hline S. No. & Mass $(\mathbf{k g})$ & Safety factor \\
\hline 1 & 0.92826 & 3.981 \\
\hline
\end{tabular}




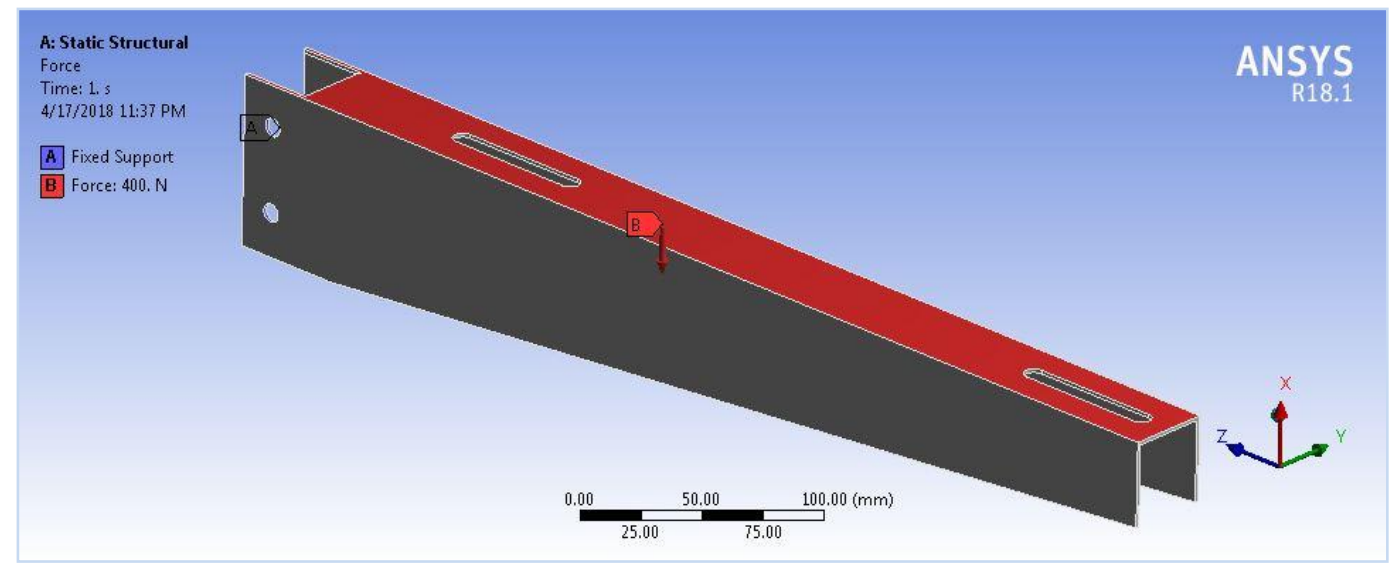

Figure 1 Load and boundary conditions of split AC condenser bracket

\subsection{Product analysis result before optimization}

The product which is supposed to be analyzed is checked for its actual ability towards satisfying the product purpose in an analytical environment where degrees of freedom and loading conditions are created in order to observe the response of the product. Figure 2 shows split AC condenser bracket safety factor for stainless steel before optimization. For considering bracket for analysis the bracket is made to react like a cantilever beam where one end of the product is applied for degrees of freedom and the other end of the product is applied for force or loading conditions and the product deformation behavior at static condition is observed. The safety factor of the product is fixed as an input parameter for optimal.

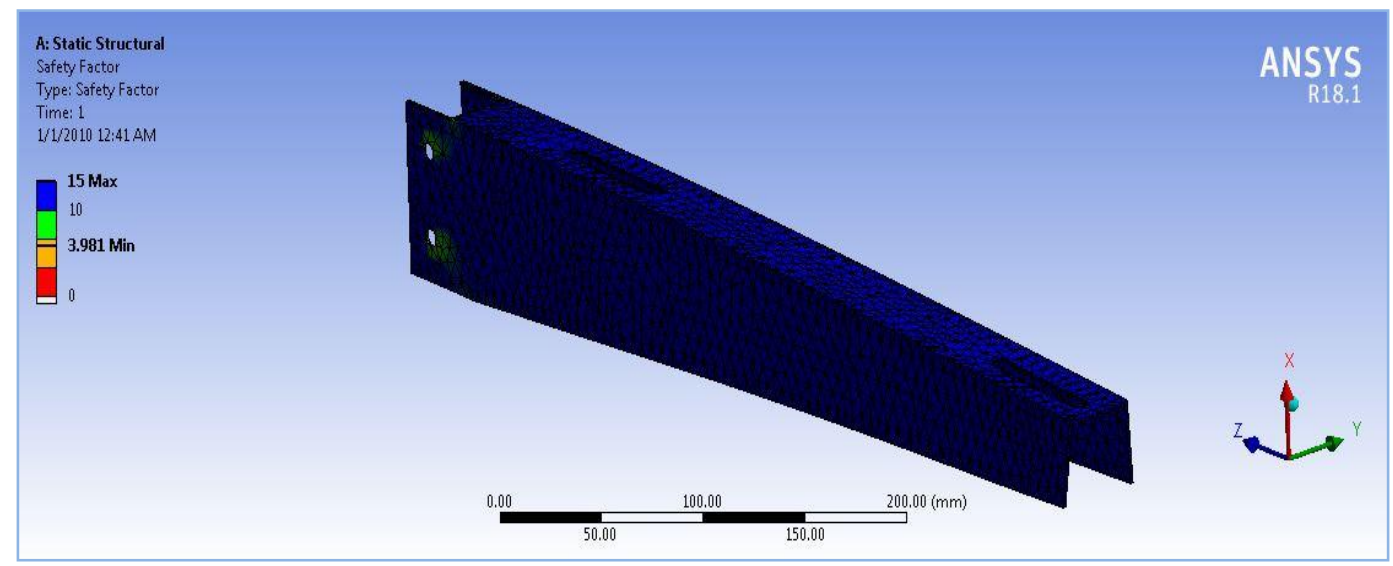

Figure 2 Split AC condenser bracket safety factor for stainless steel before optimization

\subsection{Topological optimization}

Topology optimization is a mathematical method that optimizes materials, layout within a given design space, for a given set of loads, boundary conditions and constraints with the goal of maximizing the performance of the system. Figure 3 shows the topology density of the split AC condenser bracket. The topology optimization in industries is dependent upon the purpose and the kind of work. Depending upon the purpose and the kind of work concept and design of the existing product is modified and its topology is evaluated and inspected with necessary boundary conditions. If the optimized product satisfies the boundary conditions, the product design is preceded for manufacturing process. Topology optimization of split AC condenser brackets involves the design with a constant volume within a specified boundary condition, which is to be suggested as its input parameter for attaining an optimal mass with maximum stiffness. 


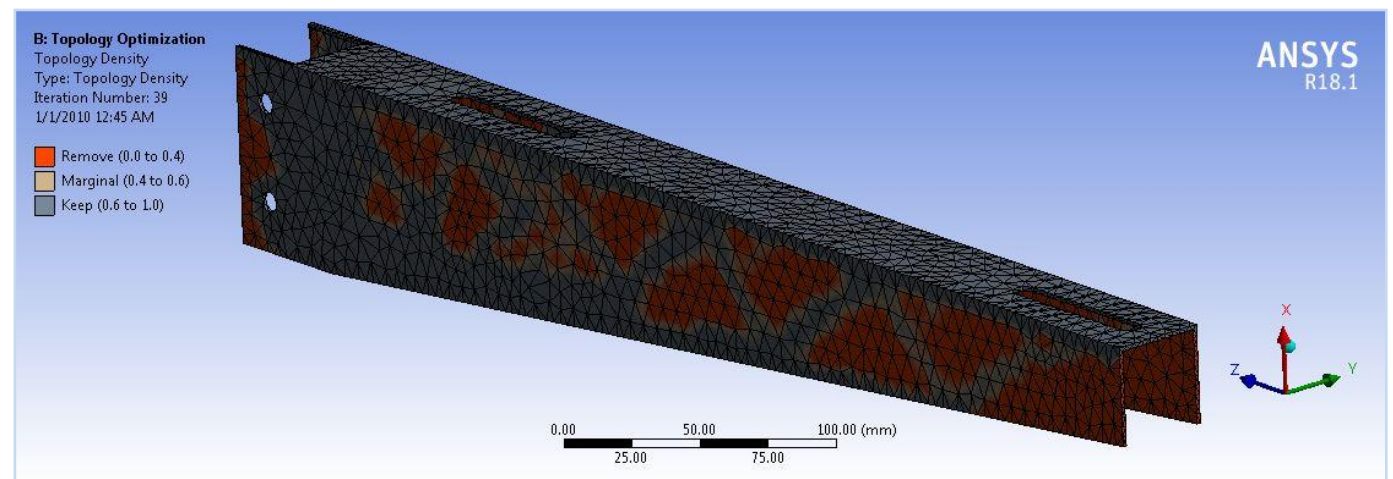

Figure 3 Topology density of split AC condenser bracket

\subsubsection{Model rebuild after refinements}

The model of the air conditioning condenser bracket is shared with topology optimization in the workbench as an interface. The necessary analytical conditions are applied for 50 percentage of product topology. The topology density result for the applied load is obtained. The obtained results are observed in a detailed manner and the optimized product topology is rebuilt with proper dimensional parameters in Creo Parametric 3.0 software. Figure 4 shows the side view of the optimized bracket model.

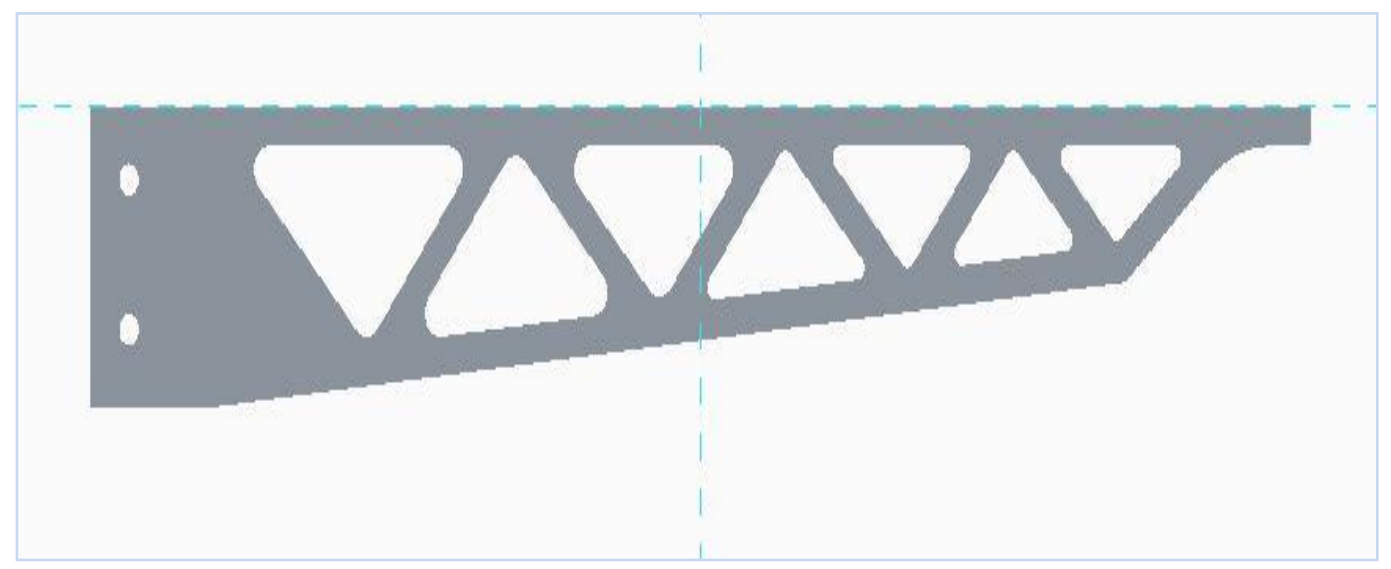

Figure 4 Side view of the optimized bracket model

\subsection{Product analysis result after optimization}

The topology of the product is modified for a fifty percentage of its topology density and the necessary analysis, such as safety factor is checked in order to get a clarity, whether the product is safe within the obtained loading conditions and parameters or not. If the results obtained where positive then further proceedings of the upcoming analysis are carried out and the obtained results are compared with the existing features of the product analytically. The split AC condenser bracket safety factor for stainless steel after optimization is shown in Figure 5. The Topology of the split AC condenser bracket after refinement is presented in Table 2.

Table 2 Topology of split AC condenser bracket after refinement

\begin{tabular}{lll}
\hline S. No. & Mass $(\mathbf{k g})$ & Safety Factor \\
\hline 1 & 0.63725 & 3.999 \\
\hline
\end{tabular}


Stalin et al.

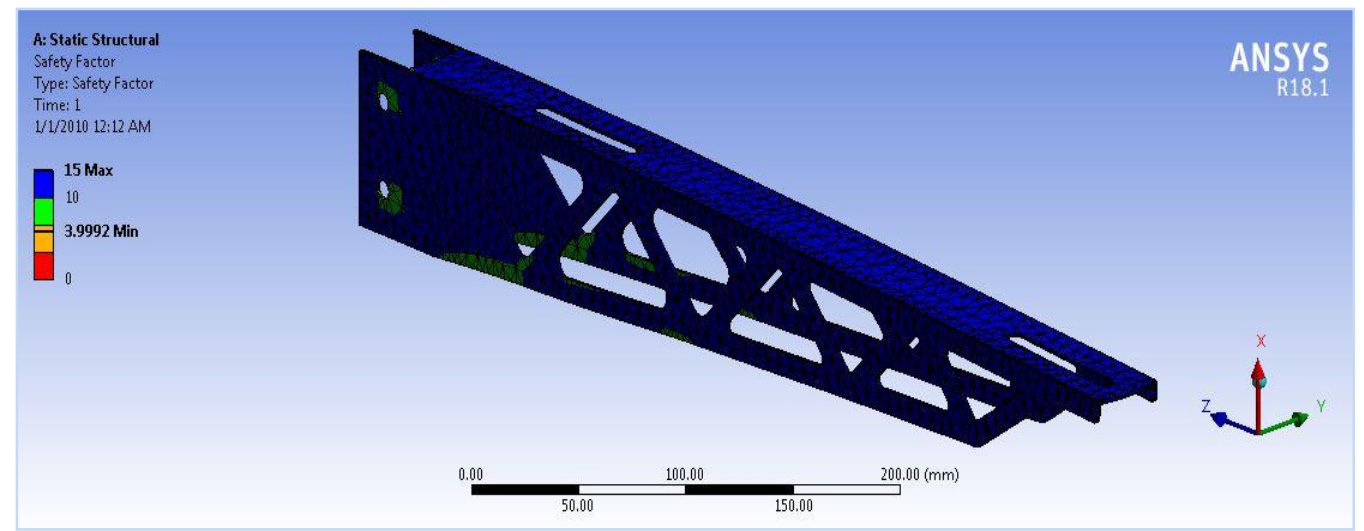

Figure 5 Split AC condenser bracket safety factor for stainless steel after optimization

\subsection{Results and comparative studies}

The topology of the split AC condenser bracket is subjected to analysis for its enhancement of factor of safety and to reduce the weight of the product. The product topology is optimized almost to half of its actual or original mass before optimization. Figure 6 shows the refinement plot for mass versus safety factor.
Figure 7 shows the model transfer into topology optimization workspace in Ansys Software. The actual weight, safety factor and volume of the product before optimization are $0.92826 \mathrm{Kg}, 3.981$ and $118250 \mathrm{~mm}^{3}$ respectively. The existing bracket model is shared into topology optimization workspace.

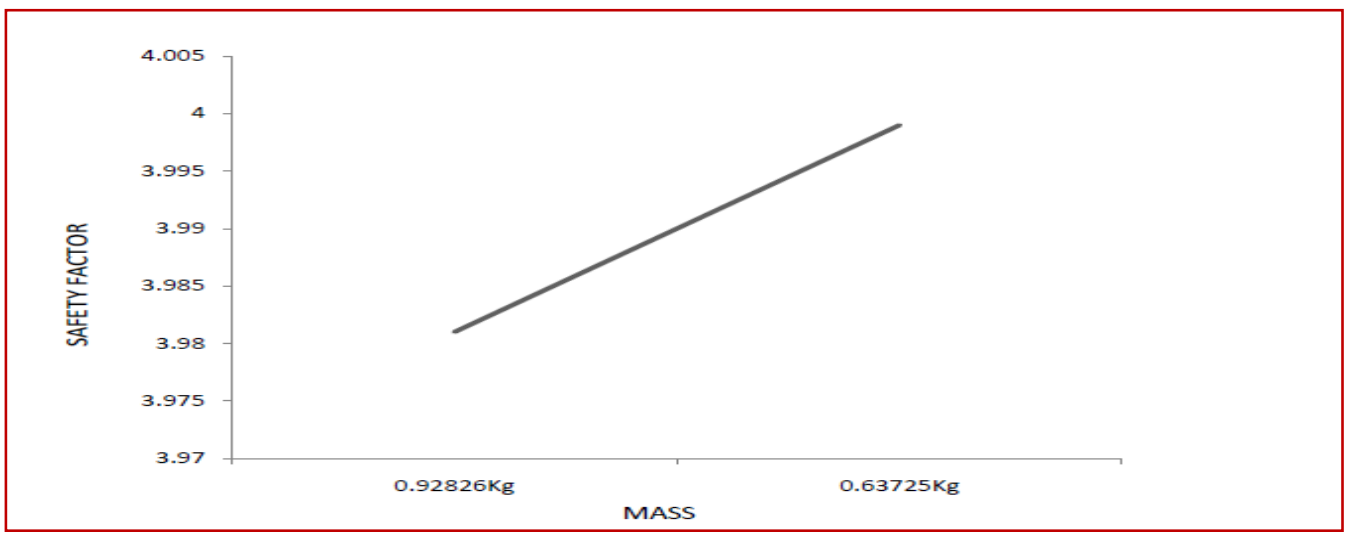

Figure 6 Refinement plot for mass vs. safety factor

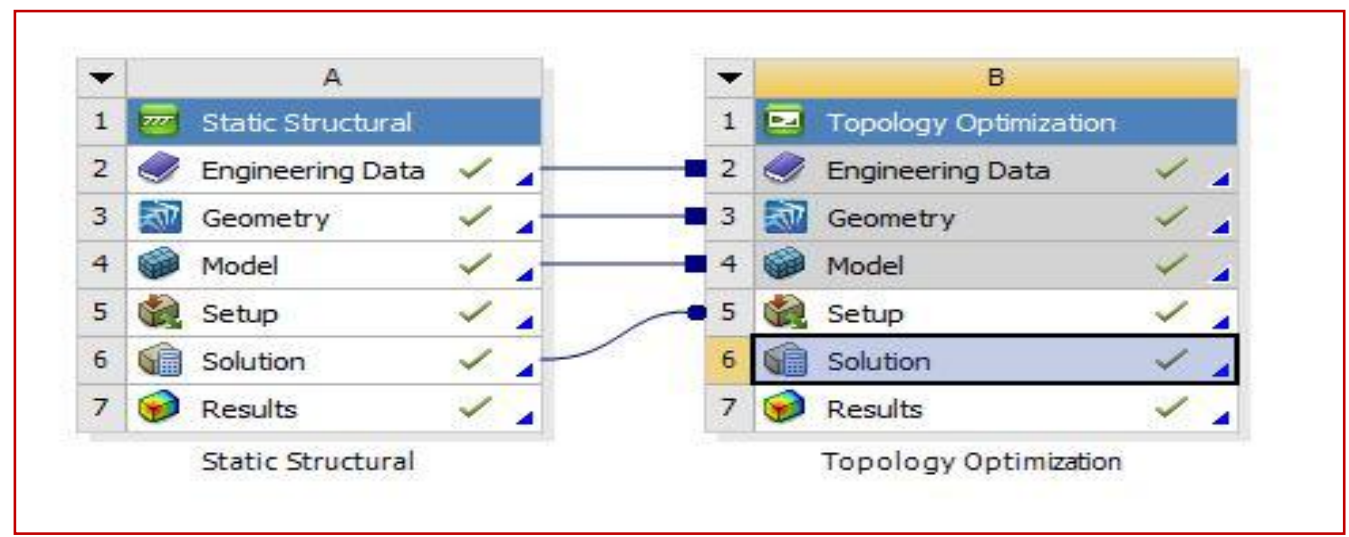

Figure 7 Model transfer into topology optimization workspace 
In order to reduce the topology density of the product, $50 \%$ topology density is kept as an input parameter. For the above suggested input value the weight and the volume of the product is $0.59209 \mathrm{Kg}$ and $75425 \mathrm{~mm}^{3}$, which is obtained as output parameter for $50 \%$ of topology density.

Based on this observation of bracket topology the bracket model is rebuild using the $3 \mathrm{D}$ modeling software and the modified bracket model is imported into the analytical environment, in order to observe the performance of the modified bracket. The weight, safety factor and volume of the product after optimization is $0.63725 \mathrm{Kg}, 3.999$ and $81179 \mathrm{~mm}^{3}$ for stainless steel materials. The comparative study of topology of split AC condenser bracket is presented in the Table 3. From the above observations the topology of split AC condenser has been enhanced without changing the actual shape and size of the product.

Table 3 Comparative study of topology of split AC condenser bracket

\begin{tabular}{llll}
\hline Optimization & Weight $\mathbf{( k g )}$ & FoS & Volume $\left(\mathbf{m m}^{\mathbf{3}}\right)$ \\
\hline Before optimization & 0.92826 & 3.981 & 118250 \\
\hline After optimization & 0.63725 & 3.999 & 81179 \\
\hline
\end{tabular}

\section{Conclusion}

Topological study of split AC condenser brackets through CAE the following observations are made,

- Based on the work conducted through analytical technique, it is observed that the weight of the split AC condenser bracket model is reduced to $68.64 \%$.

- The safety factor has improved to $0.45 \%$ of its optimum value.

Thus the obtained analytical results are satisfied for the topological design study. This methodology is applicable in automobile industry for the current trend in the design of race bikes. This study to improve the ergonomics and the aesthetic value of the products. The extension of this study, applicable in the field of Aeronautical and Automotive engineering for structural analysis of chassis structures through the CAE.

\section{Acknowledgment}

The authors thank the Department of Mechanical Engineering, Anna University, Regional Campus Madurai for providing partial support for carrying out this research work.

\section{Conflicts of interest}

The authors have no conflicts of interest to declare.

\section{References}

[1] Al-Ketan O, Rowshan R, Al-Rub RK. Topologymechanical property relationship of 3D printed strut, skeletal, and sheet based periodic metallic cellular materials. Additive Manufacturing. 2018; 19:167-83.

[2] Kazakis G, Kanellopoulos I, Sotiropoulos S, Lagaros ND. Topology optimization aided structural design: interpretation, computational aspects and 3D printing. Heliyon. 2017; 3(10).

[3] Pleshakov DV. Influence of network topology on mechanical properties of network polymers. Molecular Simulation. 2005; 31(14-15):999-1003.

[4] Lin Q, Hong J, Liu Z, Li B, Wang J. Investigation into the topology optimization for conductive heat transfer based on deep learning approach. International Communications in Heat and Mass Transfer. 2018; 97:103-9.

[5] Yang X, Li M. Discrete multi-material topology optimization under total mass constraint. ComputerAided Design. 2018; 102:182-92.

[6] Xia L, Zhang L, Xia Q, Shi T. Stress-based topology optimization using bi-directional evolutionary structural optimization method. Computer Methods in Applied Mechanics and Engineering. 2018; 333:35670.

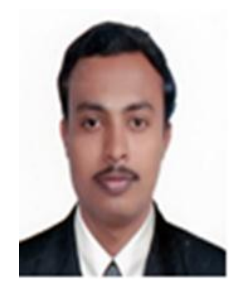

Stalin. B was born in Virudhunagar district, Tamilnadu, India, in 1981. He received B.E. Degree in Mechanical Engineering from the University of Madras, Tamilnadu, India, in 2002, and M.E. Degree in Manufacturing Engineering from the Anna University, Tamilnadu, India, in 2008. He obtained his Ph.D in Mechanical Engineering discipline, in 2015 at Anna University, Chennai, Tamilnadu, India. He has been working as an Assistant Professor in the Department of Mechanical Engineering, Anna University, Regional Campus Madurai, Tamilnadu, India since 2010. He is a Life Member of the Indian Society for Technical Education (ISTE) and The Institution of Engineers (India). His current research interests include Optimization Technology, Manufacturing Engineering, Composite Materials, Material Characterization and Mechanical Properties.

Email: stalin1312@gmail.com 
Stalin et al.



Varun Siddharth. J was born in Marudhur, Madurai district, Tamilnadu, India, in 1993. He received B.E. Degree in Mechanical Engineering from Anna University, Chennai, Tamilnadu, India in 2016. He is currently pursuing Master of Engineering in Department of Mechanical Engineering, Anna

University, Regional Campus Madurai, Tamilnadu, India since 2018. He is a Member of Society of Automobile Engineering (SAE). His current research interests in the area of Topological Optimization and Natural Fiber Composite Materials. 